\title{
Structure and tensile properties of polypropylene/carbon nanotubes composites prepared by melt extrusion
}

\author{
Yanhui Liu, Jicheng Zuo*, Jie Qin, Chengwu Li \\ College of Material Science and Engineering, Shenyang Ligong University, Shenyang, Liaoning 110168, China
}

\begin{abstract}
Polypropylene/carbon nanotubes (PP/CNTs) nancomposites were prepared with a single screw extruder by adding maleic anhydride-grafted poplypropylene (PP-g-MAH) as compatibilizer to polypropylene (PP) with different amounts of carbon nanotubes (CNTs) in the range of $0.1-0.7 \mathrm{wt} . \%$. Structure and morphology of the prepared samples were examined by Fourier transform infrared spectroscopy (FT-IR), scanning electron microscopy (SEM), polarizing light microscopy (PLM) and X-ray diffraction (XRD). The results showed that PP spherulites decreased in size when CNTs were introduced into the polymer. Mechanical properties of the samples were also studied. Tensile tests showed that with increasing amount of CNTs the strain at break decreased whereas the Young's modulus was improved of $16.41 \%$ to $36.05 \%$ and tensile strength of $36.67 \%$ to $64.70 \%$ compared to pristine PP. The SEM microphotographs showed that majority of the CNTs were dispersed individually and oriented along the shear flow direction.
\end{abstract}

Keywords: carbon nanotubes; orientation; nanocomposites; polypropylene; tensile properties

(C) Wroclaw University of Technology.

\section{Introduction}

An enormous research interest has been focused on carbon nanotubes (CNTs) since their discovery [1]. Theoretical and experimental investigations suggest that CNTs have remarkable mechanical properties with Young's modulus as high as 1.2 TPa and tensile strength of $50-200 \mathrm{GPa}$ [2]. These extraordinary mechanical properties make CNTs an ideal reinforcing filler in polymer-based nanocomposite materials. The potential of CNTs for the application as structure reinforcements depends, however, on the properties of the matrix and CNTs used and the interfacial interactions between the two materials as well as on the degree of CNTs orientation and dispersion within the matrix $[3,4]$.

Many research efforts have been directed towards producing CNTs/polymer composites for functional and structural applications $[1,5,6]$. After a decade of research, it is suggested that the method of in-situ polymerization of a monomer onto the nanotubes is the best approach for producing a nanocomposite with evenly dispersed CNTs

*E-mail: 6333787@qq.com in the matrix and providing a strong interaction between the two components at the interface [3, 7]. However, it should be noted that melt mixing and screw extruder are the most preferred methods for preparation of polymer/CNT nanocomposites for industrial applications. CNTs dispersion in nonpolar polymers such as polypropylene (PP) during melt processing has yielded mixed results. CNTs with larger diameter can be well dispersed in PP melt and the properties of the polymer-based nanocomposite materials can be improved [8-13].

In this work, polypropylene/carbon nanotubes composites were prepared by melt extrusion. At first, maleic anhydride-grafted poplypropylene (PP-g-MAH) was synthesized. Then, PP/CNTs nancomposites were prepared with a single screw extruder and by adding PP-g-MAH as compatibilizer. The variation of spherulitic morphology of PP in the nanocomposites was observed by polarizing light microscopy (PLM). To explore orientation, dispersion and cracks of the CNTs in the PP matrix, microphotographs of the nanocomposites etched with alkyl solvent along the shear flow direction and the fractured surfaces perpendicular to tensile deformation direction were subjected to detailed analysis. 


\section{Experimental}

\subsection{Materials}

PP resin (trade mark EPS30R, Qi Lu Petroleum China) with a melt flow index (MFI) of $1.83 \mathrm{~g} / 10 \mathrm{~min}$ was used as the basal polymer. CNTs were obtained from Institute of Metal Research of the Chinese Academy of Sciences. Maleic anhydride (MAH), acetone, xylene, benzoyl peroxide (BPO) and anhydrous ethanol were purchased from Sinopharm Chemical Reagent Shenyang Co., Ltd., China (chemical grade). All reagents, except BPO, were used without further purification.

\subsection{Synthesis of PP-g-MAH}

The graft copolymerization process was performed to prepare PP-g-MAH as compatibilizer by the following process: After PP and MAH were completely dissolved in xylene at an elevated temperature of $130{ }^{\circ} \mathrm{C}, \mathrm{BPO}$ as an initiator was dropwise added and reacted in nitrogen at $130{ }^{\circ} \mathrm{C}$ for $3 \mathrm{~h}$. The MAH concentration was $8 \mathrm{~g}$ per $100 \mathrm{~g}$ of $\mathrm{PP}$ resin and the mass ratio of PP to xylene was 1:5. In order to remove the unreacted monomer, the reaction products were washed in Soxhlet extractor using acetone, then dried to a constant weight at $40{ }^{\circ} \mathrm{C}$ under vacuum.

\subsection{Preparation of PP/CNTs nano- composites}

Prior to the preparation of nanocomposites, PP and CNTs were dried in a vacuum oven for $12 \mathrm{~h}$ at $80{ }^{\circ} \mathrm{C}$ to eliminate adsorbed species. PP, CNTs and PP-g-MAH were added into the mixture of solutions of absolute ethyl alcohol and acetone (the volume ratio $1: 1$ ), then the mixture was mechanically stirred for $3 \mathrm{~h}$ at a stirring speed of $180 \mathrm{rpm} / \mathrm{min}$, followed by filtration and drying. Nanocomposites were prepared using a laboratory-scale XSS-300 torque rheometer with a single screw extruder. The temperatures from the hopper to the die were maintained at $180{ }^{\circ} \mathrm{C}, 190{ }^{\circ} \mathrm{C}$ and $200{ }^{\circ} \mathrm{C}$ under continuous extrusion. The screw extruder with the length to diameter ratio of $15 / 1$ was computer-operated. The shear rate range was $20 \mathrm{rpm} / \mathrm{min}$. The molten polymer was ejected from the extruder and allowed to cool at ambient temperature. In order to prepare materials with oriented structure during packing stage, the extruded bars $80 \mathrm{~mm}$ long were put in the same direction and moulded in a hot press at $190{ }^{\circ} \mathrm{C}$, forming a $80 \times 25 \times 2 \mathrm{~mm}$ sheet, which was naturally cooled to room temperature. Formulations of the PP/CNTs nanocomposites are shown in Table 1.

Table 1. Formulations of the nanocomposites.

\begin{tabular}{cccc}
\hline Sample number & $\begin{array}{c}\text { PP } \\
\text { (wt.\%) }\end{array}$ & $\begin{array}{c}\text { PP-g-MAH } \\
\text { (wt.\%) }\end{array}$ & $\begin{array}{c}\text { CNTs } \\
\text { (wt.\%) }\end{array}$ \\
\hline \hline Pristine PP & 100 & 0 & 0 \\
PP/CNTs1 & 99.9 & 0 & 0.1 \\
PP/PP-g-MAH & 90 & 10 & 0 \\
PP/PP-g-MAH/CNTs1 & 89.9 & 10 & 0.1 \\
PP/PP-g-MAH/CNTs3 & 89.7 & 10 & 0.3 \\
PP/PP-g-MAH/CNTs5 & 89.5 & 10 & 0.5 \\
PP/PP-g-MAH/CNTs7 & 89.3 & 10 & 0.7 \\
\hline
\end{tabular}

\subsection{Characterizations and measurements}

Fourier transform infrared spectroscopy (FTIR) of the samples was recorded on a microscope FT-IR, US Nicolet 380 apparatus in transmittance mode.

PLM morphological observations of crystallites growth in the samples were performed with a Caikang polarizing light microscope. Thin films prepared from the mixture described in $2.3 \mathrm{sec}-$ tion, were inserted between two microscopic cover glasses, melted at $190{ }^{\circ} \mathrm{C}$, cooled at ambient temperature and then subjected to in situ observations.

X-ray diffraction (XRD) study was carried out in the range of $2 \theta=10-80^{\circ}$ with $\mathrm{CuK} \alpha$ radiation at a voltage of $40 \mathrm{kV}$ with a Rigaku D/maxRB (Japan) diffractometer. A current of $40 \mathrm{~mA}$ was used and the scan step was 0.02 .

Tensile tests were carried out using a tensile apparatus GB/T 1040-92. Dumbbell shaped samples of dimensions $80 \mathrm{~mm} \times 2 \mathrm{~mm}$ in the narrow section were tested using a Reger $10 \mathrm{kN}$ electronic universal testing machine (Reger. China) at a crosshead speed of $2 \mathrm{~mm} / \mathrm{min}$. For each treatment level, three 
replications were conducted. The tensile strength and modulus could directly be obtained by the provided software. The values were calculated as averages over three samples for each nanocomposition.

To clarify the orientation of CNTs, the tested samples were chemically etched in alkyl solvent. The etched surfaces were gold-coated and observed with a JEOL JSM-5900LV scanning electron microscope (SEM) under an acceleration voltage of $20 \mathrm{kV}$. The fractured surfaces of the prepared nanocomposites were also inspected by SEM in the direction perpendicular to the tensile deformation direction.

\section{Results and discussion}

\subsection{Characterization of MAH-g-PP}

Rheological and electromagnetic characterizations of the nanocomposites have demonstrated the efficiency of PP-g-MAH to promote the dispersion of CNTs in PP [13, 14]. The chemical structures of the pristine PP and MAH-g-PP have been confirmed by FT-IR in Fig. 1. In the case of PP-g-MAH, new absorption bands at 1780 and $1860 \mathrm{~cm}^{-1}$ are observed compared to pristine PP, which can be assigned to the absorption of the carbonyl groups $(\mathrm{C}=\mathrm{O})$ of MAH. The reaction products have been extracted and washed to remove unreacted MAH monomer, therefore, it is confirmed that MAH was grafted onto the PP backbone.

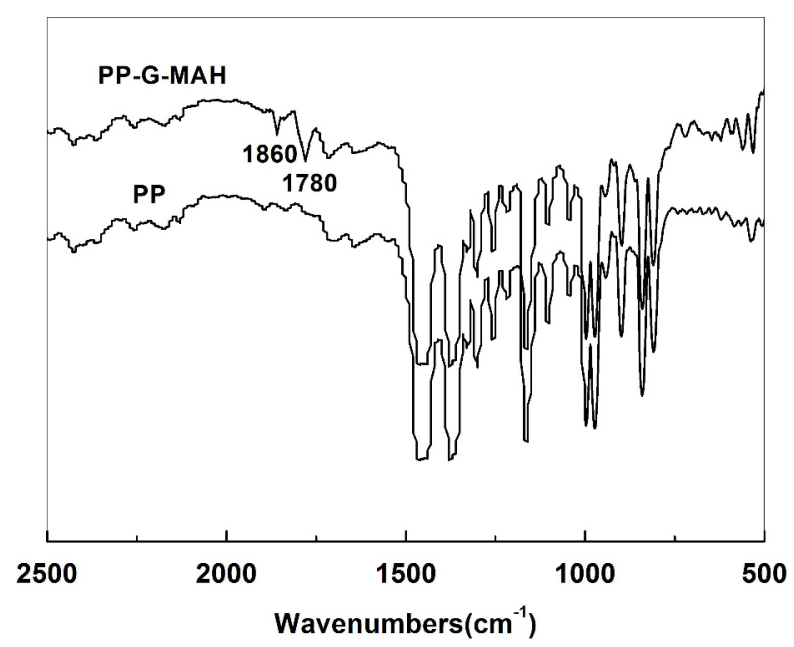

Fig. 1. FT-IR spectra of pristine PP and MAH-g-PP.

\subsection{Crystallization behavior}

It is commonly accepted that crystalline characteristics such as spherulite size and structure etc., have a profound influence on final mechanical properties of semicrystalline thermoplastics [15]. The crystallization behavior of the nanocomposites was examined by two different methods. Spherulite structure of the nanocomposites and the pristine polymer were studied at room temperature using polarizing light microscopy (PLM). The PLM pictures (Fig. 2) show smaller spherulite size (less than $40 \mu \mathrm{m}$ ) for the nanocomposites obtained with 0.5 wt. $\%$ CNTs as compared to $280-180 \mu \mathrm{m}$ for the PP-g-MAH obtained with 10.0 wt. $\%$ MAH. Pristine PP shows a regular spherulite size of 220 $160 \mu \mathrm{m}$. Decrease in spherulite size with increasing of CNTs content in PP nanocomposites has been observed earlier and can be attributed to the increased number of nucleation centers $[8,16-$ 18]. At the equivalent CNTs concentration, bigger spherulitic sizes were observed for PP/CNTs1 nanocomposites compared to that obtained after adding PP-g-MAH nanocomposites, which implies good dispersion of CNTs in the nanocomposites after adding MAH-g-PP.

The second method used was XRD. Fig. 3 shows the XRD intensity profiles of the pristine PP and the composites. The integrated XRD profiles of all the samples show five distinct reflection peaks located at $2 \theta=14.1^{\circ}, 16.8^{\circ}, 18.6^{\circ}, 21.2^{\circ}$ and $21.9^{\circ}$, associated with the diffraction of $\alpha$-form PP. No new diffraction peaks can be found with increasing CNTs content. Thus the CNTs addition has not promoted the formation of $\beta$-form PP crystals in our studies, which is inconsistent with some other studies $[16,17]$.

\subsection{Tensile properties}

The tensile properties of PP and the nanocomposites with various CNTs contents are shown in Table 2. It can be observed that the Young's modulus and tensile strength of the nanocomposites have substantially increased as compared to the pristine PP. The percentage increase in Young's modulus is from $16.41 \%$ to $36.05 \%$, and that of tensile stress achieves from $36.67 \%$ to $64.70 \%$ 


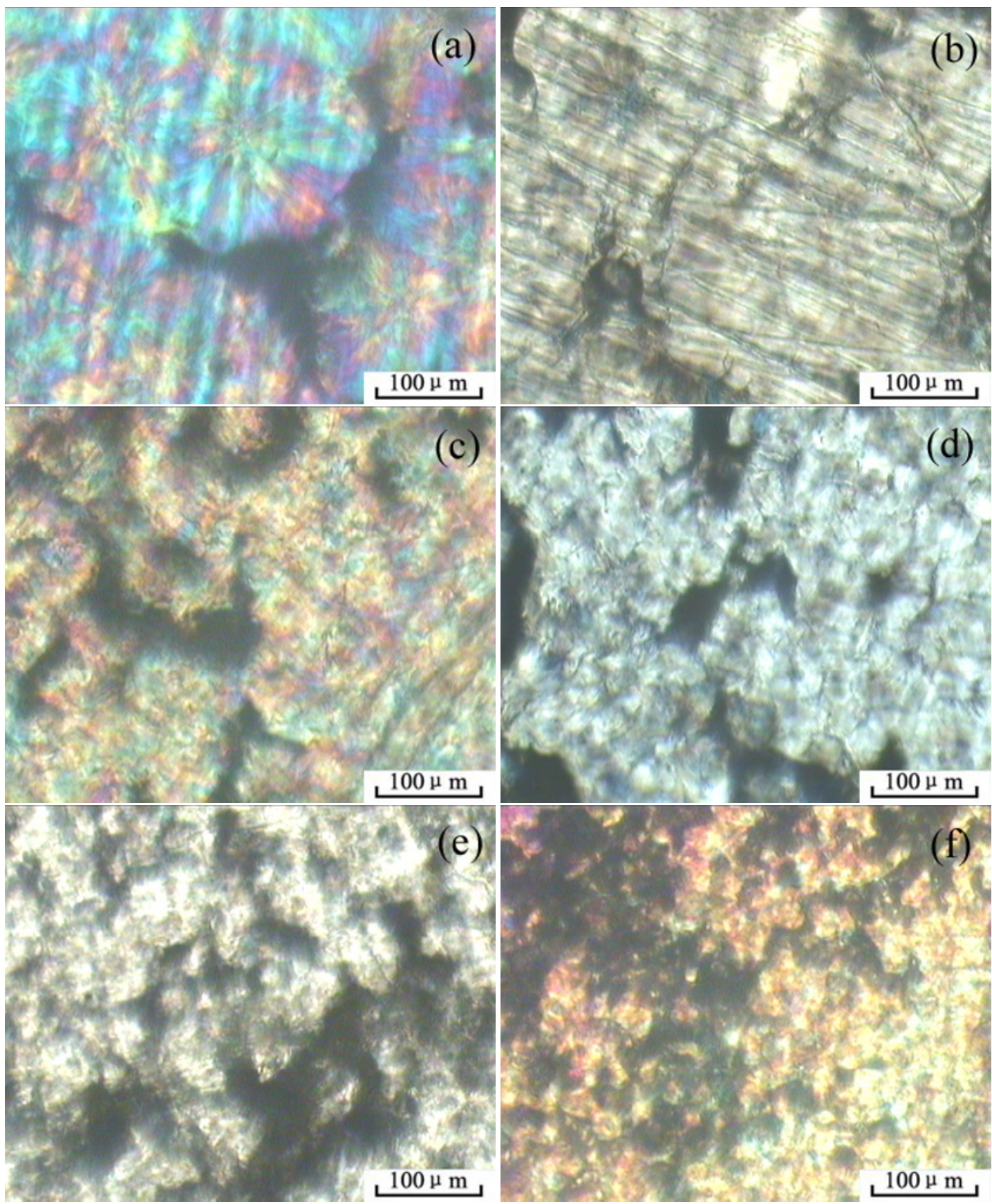

Fig. 2. PLM pictures of (a) pristine PP, (b) PP/PP-g-MAH, (c) PP//CNTs1, (d) PP/PP-g-MAH/CNTs1, (e) PP/PPg-MAH/CNTs3, (f) PP/PP-g-MAH/CNTs5.

with an increase of CNTs concentration. In case of strain at break, it decreases obviously from $42.62 \%$ to $18.77 \%$ as shown in Table 2. It means that adding CNTs can enhance the tensile strength, but the strain at break is decreased. It is due to the fact that at higher CNTs content the CNTs rich areas become more extended and tend to show interconnecting network characteristics. When such network-density becomes higher at higher CNTs concentrations it can potentially lead to intense strain localization because of hindered plastic deformation/strain of the matrix. The strain 


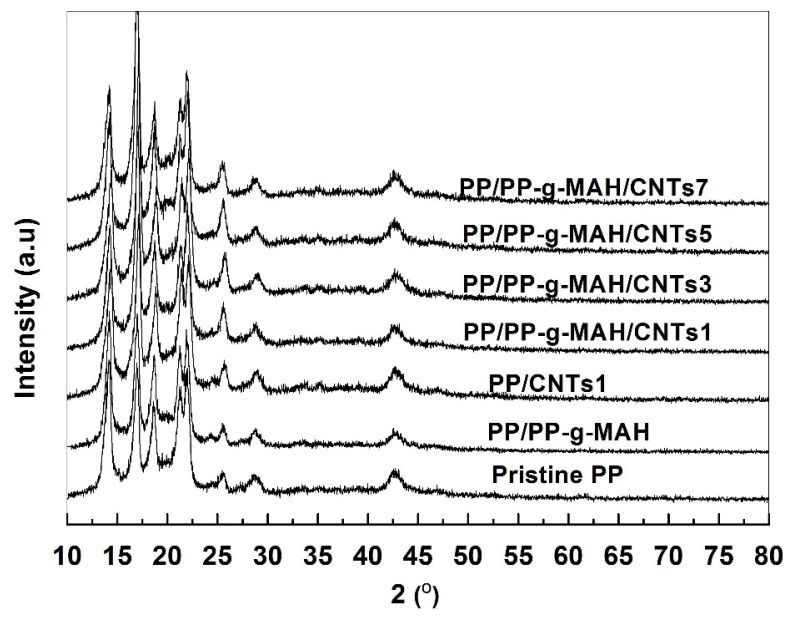

Fig. 3. XRD profiles of pristine PP, PP-g-MAH, PP/CNTs1 and PP/PP-g-MAH/CNTs with different content CNTs.

localization around the dense network of CNTs ultimately causes the matrix cracking due to severe modulus mismatch between a polymer and CNTs and hence, ductile yielding behavior is substantially reduced. Therefore, decrease in the ductility at higher CNTs content might be attributed to the presence of CNTs aggregates which act as stress concentrators. Indeed, when CNTs content increases, the number of aggregates, enlarges and as a consequence the nanocomposites become brittle. Nevertheless, the results obtained in the present studies via diluting a masterbatch are found to be similar to those obtained by direct mixing of CNTs in PP matrix as reported in the literature [19-21].

To elucidate the CNTs dispersion, an analysis of nanocomposites microphotographs has been performed and structural orientation of the samples of PP/PP-g-MAH/CNTs3 has been investigated in detail. Fig. 4a shows the SEM images of the PP/PP-g-MAH/CNTs3 nanocomposites etched with an alkyl solvent along the shear flow direction. The SEM microphotograph shows that the diameters of CNTs are around $150-250 \mathrm{~nm}$. Most of the CNTs are oriented along the shear flow direction, however, a few of the CNTs with random orientation can be observed. As shown in Fig. 4a, majority of the CNTs are dispersed individually. Nevertheless, SEM observation of tensile fracture surface also demonstrates the presence of CNTs aggregates of several micrometers (as shown by symbol $\mathrm{C}$ in Fig. 4b) which may be attributed to the imperfect mixing of CNTs with the PP. Indeed, due to the very high viscosity, the melt blending of the CNTs and the PP, even at high shear induced by a twin screw extruder, remains difficult [19]. Results of a similar study, by M. El Achaby et al. [22], using HDPE matrix, showed that some large aggregates were formed in the composites at high filler loading levels via the melt-compounding method using a minilab twin screw mixer.

The structural orientation of the CNTs can also be estimated in Fig. 4b. Majority of the broken CNTs were perpendicular to the fracture surface. Some CNTs (shown by the symbol B or A) were pulled out during the fracture of the composites.
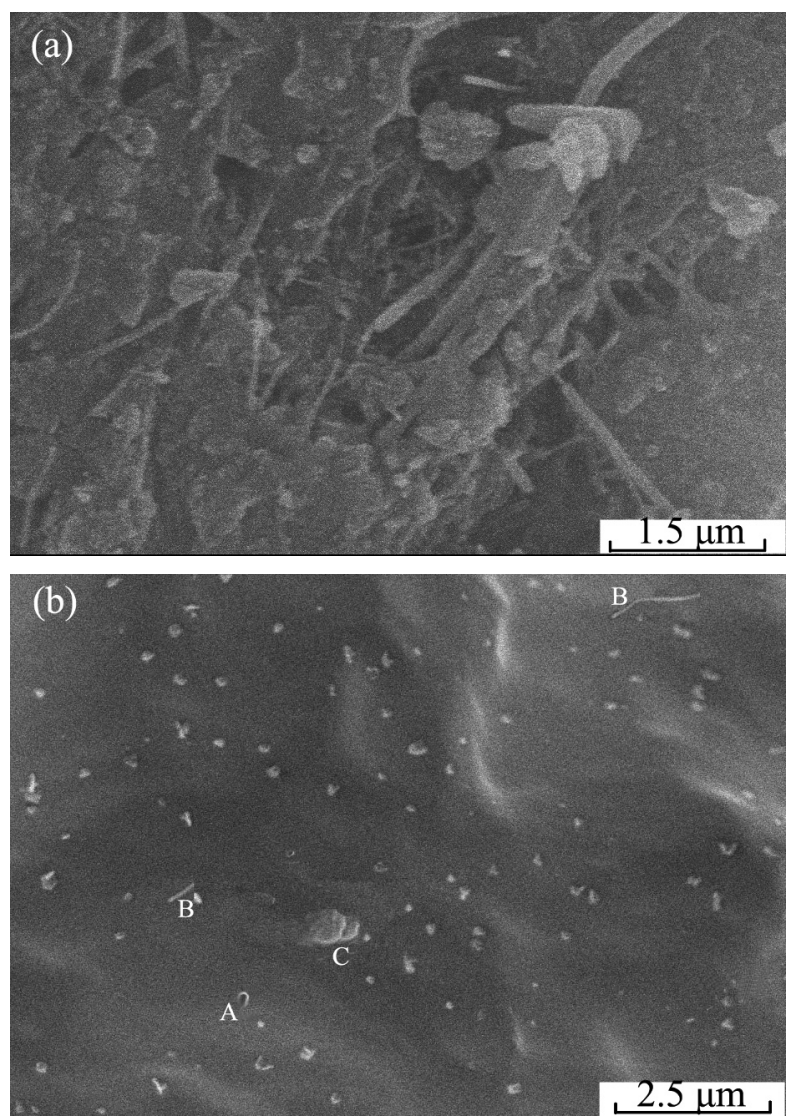

Fig. 4. SEM microphotograph of the PP/PP-gMAH/CNTs3 nanocomposites: (a) etched with an alkyl solvent along the shear flow direction and (b) the fractured surface perpendicular to tensile deformation direction. 
Table 2. Effects of CNTs content on mechanical properties of nanocomposites.

\begin{tabular}{|c|c|c|c|}
\hline Sample number & $\begin{array}{l}\text { Young's modulus } \\
\text { (MPa) }\end{array}$ & $\begin{array}{c}\text { Tensile strength } \\
\text { (MPa) }\end{array}$ & $\begin{array}{c}\text { Strain at break } \\
(\%)\end{array}$ \\
\hline Pristine PP & 220.42 & 24.08 & 42.62 \\
\hline PP/PP-g-MAH/CNTs1 & 256.58 & 32.91 & 30.06 \\
\hline PP/PP-g-MAH/CNTs3 & 272.04 & 34.52 & 22.48 \\
\hline PP/PP-g-MAH/CNTs5 & 280.69 & 35.64 & 19.52 \\
\hline PP/PP-g-MAH/CNTs7 & 299.88 & 39.66 & 18.77 \\
\hline
\end{tabular}

\section{Conclusions}

Nanocomposites based on PP and CNTs oriented along the shear flow direction could successfully be prepared with a single screw extruder using homemade PP-g-MAH as compatibilizer. The obtained nanocomposites exhibited not only improved Young's modulus, but also increased tensile strength. However, the strain at break decreased with the increasing content of CNTs. The structure and morphology of the prepared samples were examined through FT-IR, SEM, PLM and XRD. The results showed that the PP spherulites decreased in size when CNTs were introduced into the polymer. The SEM microphotographs showed that majority of the CNTs were oriented along the shear flow direction and dispersed individually.

\section{Acknowledgements}

Funding for this work was provided by Liaoning Province Education Office Foundation (L2011033) and Shenyang Municipal Science and Technology Foundation (50274061).

\section{References}

[1] Ma P.C., Siddiqui N.A., Marom G., Kim J., Compos. Part A-Appl. S., 41 (2010), 1345.

[2] Qian D., Wagner G.J., LiU W.K., YU M.F., RuO R.S., Appl. Mech. Rev., 55 (2002), 495.

[3] Razavi-Nouri M., Morteza G., Abdolhosein F., Mohsen J., Polym. Test., 28 (2009), 46.

[4] Montinaro N., Pantano A., Compos. Struct., 109 (2014), 246.

[5] Shokrieh M. M., Saeedi A., Chitsazzadeh M., Mater. Design, 56 (2014), 274.

[6] Nadia C., Adnen L., Rabeb B., Abdelaziz B., Emmanuel B., Mater. Lett., 15 (2014), 227.
[7] Sangil K., Francesco F., Hyung G P., Jung B. I., Eric M., Gabriel G., Michael S., Micha F., Jerry S., Costas P. G., Olgica B., J. Membrane Sci., 460 (2014), 91.

[8] Lee G., Jagannathan S., Chae H. G., Minus M. L., Kumar S., Polymer, 49 (2008), 1831.

[9] Huang J., Rodrigue D., Mater. Design, 55 (2014), 653.

[10] Slawomir B., JaceK G., Milo S P.S., Krzysztof K.K., Mater. Lett., 116 (2014), 53.

[11] Cheng Z.L., Kai L., Hoi M.W., Wing Y.T., Kelvin W.K.Y., SIE C.T., Mat. Sci. Eng. C-Mater., 33 (2013), 1380.

[12] Gandhi R.A., Palanikumar K., Ragunath B.K., DAVIM J.P., Mater. Design, 48 (2013), 52.

[13] Thomassin J., Huynen I., Jeromea R., DetremBLEUR C., Polymer, 51 (2010), 115.

[14] LEE S.H., Kim M.W., Kim S.H., Youn J.R., Eur. Polym. J., 44 (2008), 1620.

[15] Zhang H., Zhang Z., Eur. Polym. J., 43 (2007), 3197.

[16] Bhattacharyya A.R., Sreekumar T., LiU T., Kumar S., Ericson L.M., Hauge R.H., Smalley R.E., Polymer, 44 (2003), 2373.

[17] Liang G.D., Bao S.P., Tuong S.C., Mater. Sci. Eng. $B-A d v ., 142$ (2007), 55.

[18] Hou Z., Wang K., Zhao P., Zhang Q., Yang C., Chen D., Du R., Fu Q., Polymer, 49 (2008), 3582.

[19] Prashantha K., Soulestin J., Lacrampe M.F., Krawczak P., Dupin G., Claes M., Compos. Sci. Technol., 69 (2009), 1756.

[20] Ganb M., Satapathy B.K., Thunga M., WeIDisch R., PÖtChKe P., Jehnichen D., Acta Mater., 56 (2008), 2247.

[21] Bao S.P., TJong S.S., Mat. Sci. Eng. A-Struct., 485 (2008), 508.

[22] Аснаву M.E., QAiss A., Mater. Design, 44 (2013), 81.

Received 2014-01-09 Accepted 2014-04-10 